OPEN ACCESS

Edited by:

Georgios Kararigas,

Charité-Universitätsmedizin

Berlin, Germany

Reviewed by:

Andrea Hevener,

UCLA David Geffen School of

Medicine, United States

Elisabetta Straface,

Istituto Superiore di Sanità (ISS), Italy

Bohuslav Ostadal,

Institute of Physiology

(ASCR), Czechia

*Correspondence:

Renée Ventura-Clapier renee.ventura@u-psud.fr

Specialty section:

This article was submitted to Molecular and Structural

Endocrinology,

a section of the journal

Frontiers in Endocrinology

Received: 13 March 2019

Accepted: 29 July 2019

Published: 14 August 2019

Citation:

Ventura-Clapier R, Piquereau J, Veksler V and Garnier A (2019)

Estrogens, Estrogen Receptors

Effects on Cardiac and Skeletal

Muscle Mitochondria.

Front. Endocrinol. 10:557. doi: 10.3389/fendo.2019.00557

\section{Estrogens, Estrogen Receptors Effects on Cardiac and Skeletal Muscle Mitochondria}

\author{
Renée Ventura-Clapier*, Jérôme Piquereau, Vladimir Veksler and Anne Garnier \\ Signaling and Cardiovascular Pathophysiology Inserm UMR-S 1180, Université Paris-Sud, Châtenay-Malabry, France
}

Mitochondria are unique organelles present in almost all cell types. They are involved not only in the supply of energy to the host cell, but also in multiple biochemical and biological processes like calcium homeostasis, production, and regulation of reactive oxygen species (ROS), pH control, or cell death. The importance of mitochondria in cell biology and pathology is increasingly recognized. Being maternally inherited, mitochondria exhibit a tissue-specificity, because most of the mitochondrial proteins are encoded by the nuclear genome. This renders them exquisitely well-adapted to the physiology of the host cell. It is thus not surprising that mitochondria show a sexual dimorphism and that they are also prone to the influence of sex chromosomes and sex hormones. Estrogens affect mitochondria through multiple processes involving membrane and nuclear estrogen receptors (ERs) as well as more direct effects. Moreover, estrogen receptors have been identified within mitochondria. The effects of estrogens on mitochondria comprise protein content and specific activity of mitochondrial proteins, phospholipid content of membranes, oxidant and anti-oxidant capacities, oxidative phosphorylation, and calcium retention capacities. Herein we will briefly review the life cycle and functions of mitochondria, the importance of estrogen receptors and the effects of estrogens on heart and skeletal muscle mitochondria.

Keywords: estrogens, estrogen receptors, mitochondria, heart, skeletal muscle, sexual dimorphism

\section{CARDIAC AND SKELETAL MUSCLE MITOCHONDRIA}

\section{Mitochondria}

\section{Origin and Life Cycle}

Mitochondria are small intracellular organelles of approximately one micron in size that are present in granular or filamentary form and in variable number from one cell type to another. Mitochondria derive from aerobic $\alpha$-proteobacteria, which were engulfed by anaerobic archeobacteria giving rise to primitive eukaryotic cells a few billion years ago. The host cell provides the necessary substrates for the mitochondria that metabolize these substrates and provide ATP to the host cell through oxidative phosphorylations; in turn, mitochondria attenuate intracellular concentration of oxygen, which is a potentially toxic substance for cellular constituents.

Due to their ancient bacterial origin, mitochondria are surrounded by two membranes, an outer membrane, and an inner membrane exhibiting a particular lipid composition (rich in cardiolipin) and numerous invaginations or ridges, delimiting two spaces: the intermembrane space and the matrix space. The matrix of each mitochondrion contains several copies of a circular DNA (mtDNA) of $16 \mathrm{~kb}$ in mammals encoding 


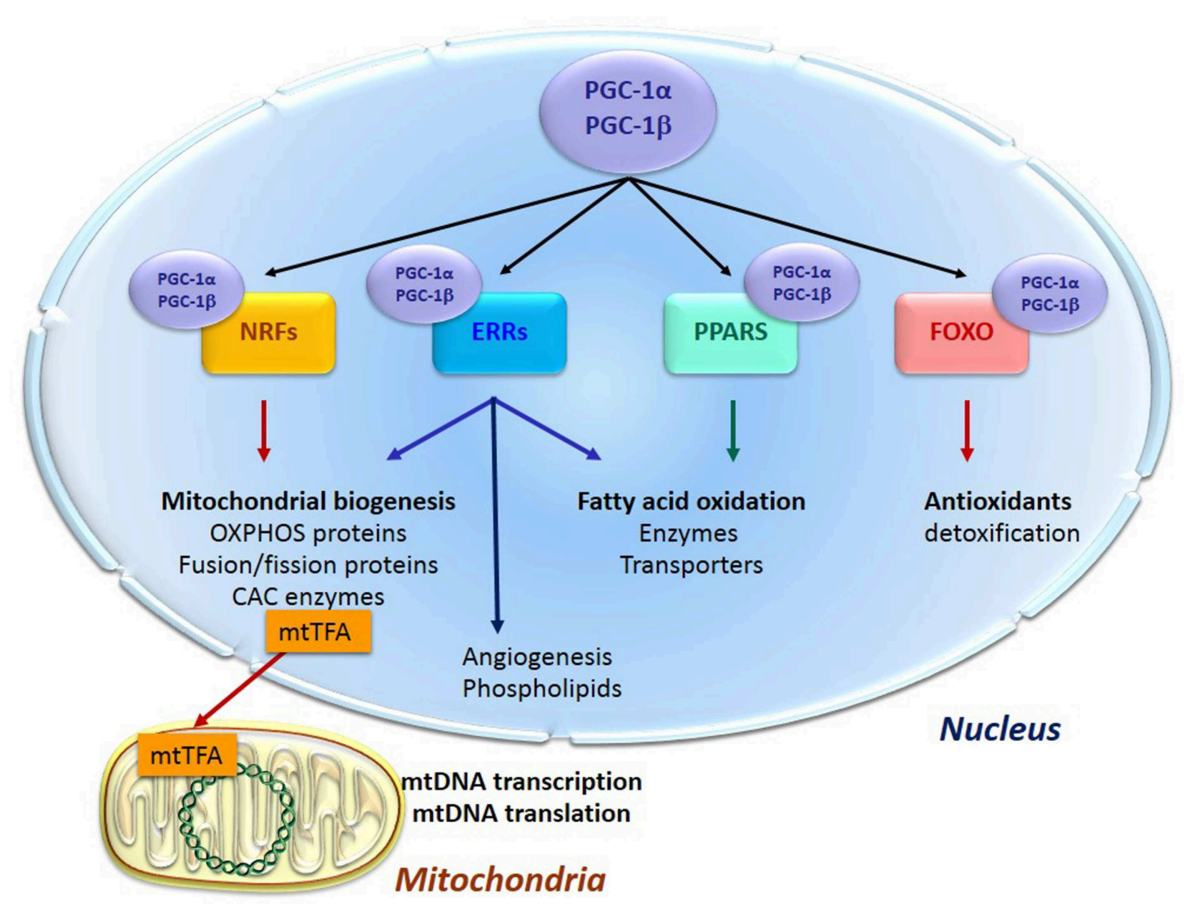

FIGURE 1 | Transcription cascade of mitochondrial biogenesis. Increased expression or activity of the master regulators of mitochondrial biogenesis "peroxisome proliferator activator receptor (PPAR) $\gamma$ co-activators" (PGC-1s) $\alpha$ and $\beta$ activate the expression of the nuclear respiratory factors 1 and 2 (NRFs) which induce the expression of the mitochondrial transcription factor A (mtTFA). The latter translocates to mitochondria, binds to mtDNA and activates its transcription and replication. PGC-1s also activate other transcription factors such as PPAR $\alpha$, mainly regulating lipid metabolism, estrogen-related receptors (ERRs) involved in substrate metabolism, energy transfer, angiogenesis and detoxification of reactive oxygen species, and the forkhead family of transcription factors (FOXOs) that activate antioxidant and detoxifying proteins.

some proteins as well as the RNAs required for the synthesis of these proteins. During evolution, mitochondrial DNA retained only 13 genes coding for subunits of the respiratory chain, two ribosomal RNA, and 22 transfer RNAs. Most of the mitochondrial genes have been transferred to the nucleus of the host cell. In mammals, the mitochondrial proteome is composed of more than a thousand of proteins (1). After fertilization, the mitochondria are of maternal inheritance. However, the fact that the vast majority of mitochondrial proteins are of nuclear origin underlies the tissue- and sex-specificity of mitochondria.

Energy supply and cell survival depend on the mitochondrial life cycle, which includes biogenesis, dynamics, and elimination

Abbreviations: CK, creatine kinase; E2, 17 $\beta$-estradiol; ER, estrogen receptor; ERE, estrogen response element; ERK1/2, extracellular signal-regulated kinase 1 and 2; ERR, estrogen-related receptors (ERRs); ETC, electron transport chain; Fis1, fission protein 1 (Fis1); GPER, G-protein coupled estrogen receptor; GSK3 $\beta$, glycogen synthase kinase $\beta$; JNK, c-Jun-NH2-terminal protein kinase; MERKO, muscle-specific ER $\alpha$ knock-out mice; Mff, mitochondrial fission factor; MFN, mitofusins; mtDNA, mitochondrial DNA; mTOR, mechanistic target of rapamycin; mtTFA, mitochondrial transcription factor A; NRF, nuclear respiratory factors; OPA1, optic atrophy 1; OVX, ovariectomy; OXPHOS, oxidative phosphorylations; p38 MAPK, mitogen-activated protein kinase; PARKIN, Parkinson juvenile disease protein 2; PCr, phosphocreatine; PGC-1, PPAR $\gamma$ coactivator; PI3K, phosphoinositide 3-kinase; PINK1, PTEN-induced putative kinase 1; PKB, protein kinase B; PPAR, peroxisome proliferator activator receptor (PPAR) $\gamma$ co-activators; PTP, permeability transition pore; ROS, reactive oxygen species; SERM, selective estrogen receptor modulator. of defective organelles by mitophagy. Mitochondrial proliferation consists of growing of preexisting organelles, through a process called mitochondrial biogenesis followed by mitochondrial division (fission). This biogenesis requires the coordination of both the nuclear and the mitochondrial genomes; it is mostly under the control of the nucleus through transcriptional cascades involving coactivators and specific transcription factors (Figure 1). Among the most important are (1) the family of the transcriptional coactivators "peroxisome proliferator activator receptor (PPAR) $\gamma$ co-activators" (PGC-1s) $\alpha$ and $\beta$, the master regulators of mitochondrial biogenesis, (2) nuclear respiratory factors NRF1 and two involved in the nuclear control of mitochondrial biogenesis (2), (3) transcription factors such as $\operatorname{PPAR} \alpha$, mainly regulating lipid metabolism, (4) estrogen-related receptors (ERRs) involved in substrate metabolism, energy transfer, angiogenesis, and detoxification of reactive oxygen species and (5) the mitochondrial transcription factor A (mtTFA) which once synthesized, migrates to the mitochondrial matrix where it activates the transcription and replication of mtDNA.

An important step in the mitochondrial life cycle is the fission of mother mitochondria into two daughter mitochondria, which is coordinated by specialized GTPases. In most cells, mitochondria are organized into a network that undergoes frequent fusion and fission events. This process is called mitochondrial dynamics. Fission is dependent on the DRP1 protein and its docking proteins, fission protein 1 (Fis1) and 
mitochondrial fission factor (MFF). Fusion is controlled by mitofusins (MFN1 and 2) for the outer membrane and optic atrophy 1 (OPA1) for the inner membrane. These fusion/fission processes are very slow in the adult cardiomyocyte under normal conditions (3).

Finally, dysfunctional mitochondria are eliminated through a specific mechanism of autophagy called mitophagy. Autophagy is the process by which cellular components such as macromolecules and organelles are recycled, allowing for the elimination of cellular waste and the reconstitution of nutrient stores. Mitophagy is the specific process for elimination of mitochondria and it is involved in quality control and mitochondrial turnover rate regulation. This process involves many proteins, among which the E3-ubiquitin ligase Parkinson juvenile disease protein 2 (PARKIN) and the PTEN-induced putative kinase 1 (PINK1) can be mentioned, allowing the identification of damaged mitochondria and their addressing to the autophagosome, the latter subsequently fusing with the lysosome to form autophagolysosomes.

\section{Mitochondrial Functions and Regulation}

Mitochondria are the main site of transformation of the energy of different substrates into chemical energy in the form of ATP. ATP consumption is extremely high in the heart where mitochondria provide more than $90 \%$ of the energy needed for contraction and cell pumps. The linear relationship between oxygen consumption and cardiac work shows that the mitochondria work in a "pay as you go" manner in this tissue. The heart can use a variety of substrates to regenerate ATP according to their availability, mainly fatty acids. While slow oxidative muscles also largely depend on oxidative metabolism for ATP production and uses fatty acids and glucose, fast skeletal muscles function in a "work first pay later" manner and preferably use glucose, glycogen and glycerol-3P. Lipid oxidation is responsible for 60 and $80 \%$ of totally consumed oxygen by soleus and cardiac muscle mitochondria, respectively, but for only a few percent by glycolytic gastrocnemius muscle mitochondria. On the opposite, glycerol-3-P utilization represents more than $80 \%$ of substrate used by gastrocnemius muscle mitochondria, while $<10 \%$ of substrates used by cardiac mitochondria (4).

The common degradation product of lipids, amino acids and carbohydrates in mitochondria is acetyl-CoA, which enters the Krebs cycle in the mitochondrial matrix resulting in the production of reducing equivalents $\left(\mathrm{NADH}\right.$ and $\left.\mathrm{FADH}_{2}\right)$. These reducing equivalents are re-oxidized by the electron transport chain (ETC), also called respiratory chain, through the process of oxidative phosphorylations (OXPHOS). The respiratory chain is composed of four complexes forming a redox chain which results in the reduction of molecular oxygen to water and the creation of a proton gradient across the mitochondrial inner membrane. This electrochemical gradient of protons serves as a protonmotive force to rephosphorylate ATP from ADP by ATP synthase considered as complex V of ETC. Mitochondrial respiration is controlled by ADP according to a Michaelis/Menten relationship (5) and is modulated by calcium through the regulation of the activity of certain enzymes in the Krebs cycle and the respiratory chain (6). ATP is exchanged for ADP by the adenine nucleotide translocator anchored in the inner membrane. In muscle and cardiac cells, most of the ATP produced is immediately transformed in the inter-membrane space into phosphocreatine (PCr) by mitochondrial creatine kinase (CK) located on the outer surface of the inner membrane in the reaction:

$$
\mathrm{ATP}+\text { creatine } \Leftrightarrow \mathrm{ADP}+\mathrm{PCr}+\mathrm{H}^{+} .
$$

The newly formed PCr is channeled to the sites of energy utilization by the cytosolic CKs and allows the rephosphorylation of ADP locally produced by the ATPases $(5,7)$.

In addition to providing the greatest amount of energy to the host cell, mitochondria participate in other cellular functions such as ionic homeostasis among which calcium plays a pivotal role, production and regulation of reactive oxygen species (ROS), $\mathrm{pH}$ control and cell death. Besides, mitochondria are crucial for steroid hormone synthesis, thermogenesis, and lipid and carbohydrate utilization in a tissue-specific manner.

Mitochondria are the main site of ROS production in the cells and also the primary target of oxidative damages. ROS are mainly produced at the level of complex I and complex III of the respiratory chain. In physiological conditions, mitochondrial and cytosolic anti-oxidant defenses allow to control any excess of ROS production. Dysregulation of ROS production is involved in many pathological situations and in aging.

Another critical factor in mitochondrial function is the regulation of intracellular and intramitochondrial calcium. Although calcium is known to activate mitochondrial dehydrogenases, when present in excess, it uncouples mitochondria and decreases ATP production. High calcium collapses the mitochondrial membrane potential and induces the opening of the permeability transition pore (PTP), triggering the liberation of proapoptotic factors and leading to cell death (8).

\section{Tissue Specificity of Mitochondria}

Quantity, composition and function of mitochondria are adapted to the tissue in which they are embedded (9). It is generally believed that tissues with high and sustained ATP demand like striated muscles depend on oxidative phosphorylations ensured by the mitochondria. However, striking differences exist between oxidative cardiac and skeletal muscles on one hand and glycolytic skeletal muscles on the other hand. Cardiac muscle and postural skeletal muscles are strictly dependent on oxidative ATP production as they have to sustain long-term contractile activity. Oxidative muscles should have accurate adjustment of energy production to energy consumption. This is different from fast skeletal muscles whose contractile activity is rapid and short in duration and is mainly supported by ATP and PCr stores and glucose- or glycogen-driven ATP production.

In the myocardium, one of the most oxidative tissues of the body, mitochondria occupy $30-40 \%$ of the cell volume against $6-8 \%$ in the slow and $2-3 \%$ in the fast muscle fibers. The mitochondria in oxidative tissues like the heart and slow oxidative postural skeletal muscles are more numerous, located largely near the myofilaments, and able to use essentially fatty acids. They have a relatively low sensitivity to ADP and a significant amount of mitochondrial CK allowing them to 
participate in intracellular energy transfer shuttles. They are adapted to the continuous supply of energy, necessary for muscular endurance work and cardiac function (10).

In fast skeletal muscles, mitochondria are less numerous, with a high sensitivity to cytosolic ADP (11) and are more dependent on glycolysis-derived substrates such as pyruvate and glycerol-3P (4). The energy required for the phasic activity of fast muscles is in fact essentially dependent on anaerobic glycolysis; energy metabolism reflects rapid and short-term use of large amounts of cytosolic ATP and PCr. Importantly, the CK system also exhibits important tissue specificity among striated muscles (9).

\section{Sexual Dimorphism of Mitochondria}

Although mitochondria are of maternal inheritance in both males and females, it should be kept in mind that, as discussed above, almost all mitochondrial proteins are encoded by the nucleus and thus under the influence of sex chromosomes, epigenetic marks and importantly, circulating sexual hormones. It is thus not surprising that in addition to a clear tissue specificity of mitochondria, a sexual dimorphism of mitochondria has been observed and starts to be studied in further details. Evidence is accumulating that this dimorphism takes part in the sex-specificity of important pathologies (12-14). Mechanistic pathways involved in sex differences in cardiovascular diseases has been recently reviewed in depth (15).

\section{Cardiac Muscle}

Gene expression profiling of mouse and human cardiac samples revealed sex and aged dependent expression patterns $(16,17)$. Among the differences, energy metabolism pathways are wellrepresented. However, it is generally accepted that cardiac oxidative capacity does not essentially differ between males and females (18-20) except for fatty acid utilization capacity that seems to be higher in young females, and for oxidative capacity that declines slower with age in older females $(21,22)$.

The most striking difference between male and female mitochondria lies in antioxidant defenses $(12,23)$. Female cardiac mitochondria seem to produce less $\operatorname{ROS}(18,20)$ although this may be species or strain-dependent (19). Decreased ROS generation in female rat cardiac mitochondria has been attributed to posttranslational modifications of mitochondrial proteins involved in ROS handling (24).

Cardiac mitochondria of female rats have a greater calcium retention capacity than the male ones (25). Calcium kinetics also differ between male and female rat cardiac mitochondria (26). Mitochondria from females are more resistant to mitochondrial swelling at high calcium concentration (27). The lower $\mathrm{Ca}^{2+}$ uptake rates and the maintenance of mitochondrial membrane potential under conditions of high $\mathrm{Ca}^{2+}$ in female rat cardiac mitochondria have been attributed to modulation of the calcium uniporter (28).

Thus, sexual dimorphism of cardiac mitochondria involves a lower production of ROS, higher antioxidant defenses, and a better regulation of intra-mitochondrial calcium; all of which participate in the better resistance of female cardiomyocytes to ischemia/reperfusion injury, heart failure or cardiotoxicity.

\section{Skeletal Muscle}

It is well-established that female rodents have much higher endurance capacities than their male counterparts $(29,30)$. Interestingly woman skeletal muscle demonstrates an increased fatigue resistance compared to man muscle (31). Endurance capacity is highly dependent on skeletal muscle mitochondrial content and quality, as well as capacity to oxidize lipid substrates. Compared to males, females rely to a greater extent on fat oxidation during exercise (32) and skeletal muscles of women have higher intracellular lipid content than male muscles (33). Moreover, gastrocnemius muscle of female rats exhibits higher mitochondrial mass, oxidativephosphorylative capacities, and mtDNA/nDNA compared to males $(34,35)$.

Different lean mass/body mass ratio in male and females, and the plasticity of skeletal muscle mitochondria in response to exercise training and exercise capacity (36), make comparison of skeletal muscle from men and women more difficult to interpret than for laboratory animals which are usually kept in standard low activity conditions. Recent studies have brought new knowledge on sex differences in human skeletal muscle mitochondria $(37,38)$. Whether these differences are due to differences in skeletal muscle composition in terms of oxidative vs. glycolytic fibers, remain to be determined. In young men and women of similar aerobic exercise capacity, a higher mitochondrial volume density and increased capacity for fatty acid and lactate oxidation were found in women skeletal muscle (39) thereby suggesting real differences between men and women mitochondrial properties.

Other aspects of sex differences in mitochondria include resistance to calcium overload, generation of radical oxygen species, and susceptibility to PTP opening and apoptosis. Only few data are available for skeletal muscle.

Thus, mitochondria of skeletal muscle also seem to exhibit a sexual dimorphism which could explain the higher facility of females to adapt to altered metabolic energy situations. However, data on skeletal muscle are so far sparse and undoubtedly, sexual dimorphism should be investigated more systematically in order to know whether skeletal muscle mitochondria contribute to different men and women muscle function and susceptibility to pathologies (13).

How much of these effects could be attributed to sex hormones and more specifically to estrogens and estrogen receptors will be discussed below.

\section{ESTROGEN RECEPTORS AND MITOCHONDRIA}

Estrogen effects are mainly mediated through estrogen receptors (ERs). ERs belong to the family of steroid hormone receptors themselves members of the family of ligand-activated transcription factors. Three estrogen-receptors have been described, estrogen-receptor alpha $(\mathrm{ER} \alpha)$, estrogen receptor beta $(\mathrm{ER} \beta)$, and the G-protein coupled estrogen receptor (GPER) with specific tissue and intracellular locations (Figure 2). These receptors are thought to be present in practically all cell types in 


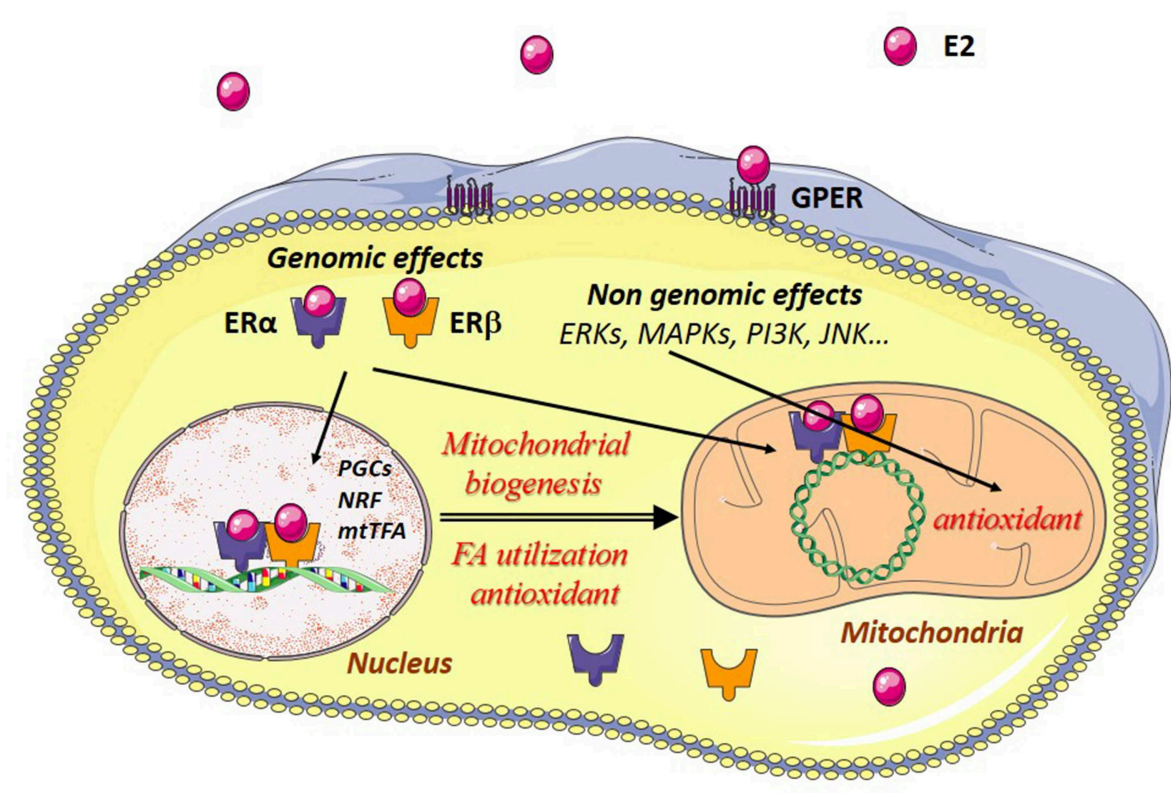

FIGURE 2 | Estrogen effects in muscle cells. Estradiol (E2) binds to its cytosolic receptors, estrogen-receptor $\alpha(E R \alpha)$ and $\beta$ (ER $\beta)$ inducing the translocation of the complex E2/estrogen receptors (ER) to the nucleus. Interaction of this complex with nuclear DNA results in the transcription of Pgc-1 $\alpha$, Nrfs, and other mitochondrial genes are involved in mitochondrial biogenesis, fatty acid utilization, and antioxidant defenses. Estradiol also interacts with ERs bound to mitochondrial DNA (mtDNA), thereby leading to transcription and replication of the mtDNA. E2 can also bind to G-protein-coupled estrogen receptors (GPER) at the plasma membrane, activating intracellular signaling pathways like extracellular signal-regulated kinase 1 and 2 (ERK1/2), p38 mitogen-activated protein kinases (MAPKs), phosphoinositide 3-kinase (PI3K), c-Jun-NH2-terminal protein kinase (JNK), and others. This results in the transcription of genes encoding antioxidant enzymes, thus reinforcing in particular the antioxidant defenses of the mitochondria.

varying quantities. Their regulation and their location in striated muscles are still not well-understood.

Estrogens may have genomic and non-genomic effects. ERs may function as nuclear receptors and transcription factors in the nucleus and as signaling molecules in the plasma membrane. Estrogens regulate nuclear gene transcription by binding and activating the classical genomic estrogen receptors $\alpha$ and $\beta$ $(\mathrm{ER} \alpha$ and $\mathrm{ER} \beta)$ and by activating plasma membrane-associated ERs, and GPER. The major circulating estrogen is $17 \beta$-estradiol (E2) which equally binds ER $\alpha$ and ER $\beta$. The genomic effects involve dimerization of two ERs upon binding of E2 which then translocate to the nucleus and bind to estrogen response elements (ERE), thus regulating specific gene expression. Binding of $\mathrm{E} 2 / \mathrm{ER} \alpha$ complex to estrogen response elements (EREs) is regulated by phosphorylation independent of ligand binding. For detailed description of the genomic effects of E2/ER see (40).

Non-genomic effects involve rapid, within seconds or minutes, signaling actions through activation of membraneassociated ERs and GPER and subsequent activation of signaling pathways like extracellular signal-regulated kinase 1 and 2 (ERK1/2), increased phosphorylation of c-Jun-NH2-terminal protein kinase (JNK), phosphoinositide 3-kinase (PI3K), protein kinase $B(P K B)$, glycogen synthase kinase $3 \beta$ (GSK3 $\beta), \beta$-catenin, calcineurin, mechanistic target of rapamycin (mTOR), p38 mitogen-activated protein kinase (MAPK), and others $(15,41)$.
Both $E R \alpha$ and $\operatorname{ER} \beta$ were found in male and female cardiomyocytes $(42,43)$. However, one study showed transcripts only for $\mathrm{ER} \alpha$ in isolated cardiomyocytes from neonatal or adult rats suggesting that ER $\beta$ could be present in other cardiac cell types like fibroblasts or vascular cells (44). The presence of both ERs in cardiomyocytes is thus a matter of debate while skeletal muscles express both ERs with certainty $(45,46)$.

In addition to their presence in nuclei and plasma membrane, both $\operatorname{ER} \alpha$ and $\operatorname{ER} \beta$ have been detected in mitochondria of many cell types and species [for details see recent reviews (40, 47-49)]. However, ER $\beta$ seems to be the main estrogen receptor present in mitochondria as demonstrated by immunohistochemistry, immunocytochemistry, and immunoblots using a large panel of antibodies and mechanisms of import have been studied (see (50) for review).

Mitochondrial location of E2 receptors seems to be anyway tissue-specific. The presence of ER $\beta$ was detected in human heart mitochondrial proteins by Yang et al in 2004 (51) while not detected in liver cells by the same methods (MALDITOF) (52). ER $\beta$ was also identified in the skeletal muscle tissue and the $\mathrm{C} 2 \mathrm{C} 12$ skeletal muscle cell line (53, 54). In this cell line, ER $\beta$ interacts with the chaperone HSP27 in the mitochondria and stabilizes it (55). However, additional experiments are needed to clearly identify the tissue specific expression and role of the different estrogen receptors in mitochondria. 


\section{ESTROGEN EFFECTS ON MITOCHONDRIA}

The effects of estrogens on mitochondria are not restricted to their presence and role inside mitochondria. As discussed above, expression of mitochondrial proteins is mainly controlled by nuclear genome so that nucleus controls mitochondrial biogenesis. It is thus not surprising that the major effects of estrogen on mitochondria arise from their nuclear effects.

Estrogens exert a protective role on mitochondria by direct and indirect effects in a number of tissues [reviewed in $(40,48)$ ]. Estrogen regulation of mitochondrial mass and function has been shown to participate in vascular, cardiac and neuronal protection (56-59). Estrogens appear to modulate various aspects of mitochondrial function including ATP production, ROS generation, antioxidant defenses, mitochondrial membrane potential, and calcium handling (50). Moreover, estrogens via ERs are involved in life cycle of mitochondria controlling mitochondrial biogenesis, mitochondrial quality control, and mitophagy. These effects may be mediated by both genomic and non-genomic effects.

Estrogens favor mitochondrial biogenesis in a tissuespecific manner by stimulating or inhibiting the expression of mitochondrial proteins from both nuclear and mitochondrial genomes $(48,60)$. Estrogens directly modulate the expression of mitochondrial protein genes by binding of ER/E2 to the ERE of metabolic genes in the nucleus. They increase the expression of the master regulator of energy metabolism and mitochondrial biogenesis PGC- $1 \alpha$ and its downstream targets $(61,62)$. E2 also stimulates mitochondrial biogenesis by ER/E2 mediated activation of the transcription factors NRFs (56, 62, 63). NRF1 increases transcription of mitochondrial nuclear-encoded genes and of mitochondria-encoded genes through increased production of the transcription factor mtTFA (64). This is mediated by ER $\alpha$ and by the presence of an ERE in the promoter of the NRFs which can bind both ER $\alpha$ and ER $\beta$ in an estrogen-dependent manner (63).

ERs may also bind to mitochondrial DNA and are involved in the E2-induced expression of mtDNA and respiratory chain proteins, suggesting a role for these receptors in the regulation of mtDNA transcription and replication. The presence of ERs and their effects on both the nuclear and mitochondrial genomes would ensure their coordination in response to estrogens.

Whether ER $\alpha$ and ER $\beta$ exert similar or dissimilar roles in a tissue-specific manner is an additional level of complexity. Indeed, in addition to their subcellular localization, estrogen action depends on the expression and content of selective estrogen receptor modulators (SERMs). These SERMs induce various conformational changes in the ERs and differential recruitments of corepressors, coactivators, and transcription factors so that they can be either full or partial agonist or antagonists (47).

Thus, E2 is able to directly or indirectly modulate mitochondrial biogenesis and mitochondrial properties. The implication and role of ER $\alpha$ and/or ER $\beta$ may be tissue specific.

\section{Estrogen Effects on Cardiac Mitochondria}

Several studies have described the effects of ovariectomy on cardiac mitochondria. Quantitative proteomic analysis of cardiac mitochondria from ovariectomized (OVX) aged rats revealed a remarkable reduction in mitochondrial proteins involved in ETC, oxidative phosphorylation, lipid, and carbohydrate metabolism as well as oxidative stress and apoptosis (65). Ovariectomy decreases the activity of ETC complexes, increases mitochondrial membrane lipid peroxidation, and decreases calcium handling capacity (66). It decreases complex I-driven ATP synthesis and increases stress-induced ROS production of isolated cardiac mitochondria (58). Ovariectomy also downregulates cardiac mitochondrial biogenesis and function markers and increases oxidative stress through a possible GPER-mediated effect (67). These effects could be counteracted by E2 treatment. For example, E2 increases NRF-1, TFAM, PGC-1 $\alpha$, and mitochondrial protein levels in the heart of OVX mice (68). Mitochondrial alterations due to E2 deficiency may also be caused through miR-23a-mediated PGC- $1 \alpha$ downregulation, which may subsequently be involved in the menopauseassociated concentric cardiac hypertrophy (69).

On the other hand, physiological concentrations of estrogens do not affect mitochondrial respiratory functions in normal conditions but protect heart mitochondria from high calciuminduced release of cytochrome $c$ (70). Activation of the membrane receptor GPER provides a positive effect after ischemia-reperfusion injury by inhibiting PTP opening, an effect mediated by the ERK pathway (71). E2 via ER $\beta$ mediates cardioprotection following trauma hemorrhage by activating the transcriptional cascade of mitochondrial biogenesis (62) and inhibiting the mitochondria-mediated apoptotic pathway (72). ER $\beta$ also appears to mediate the anti-apoptotic effects of estrogen in female adult heart following pressure overload (73). In cardiomyocytes exposed to ischemia/reperfusion, estrogens prevent cardiomyocyte apoptosis by inhibiting mitochondrial ROS formation (74). ER $\beta$ also mediates the increased expression of key regulators of cardiac mitochondrial function and respiratory chain proteins occurring in female mice following voluntary running (30). Thus, effects of E2 on mitochondria participate in the regulatory effects of estrogens. Finally, cardiomyocyte-specific deletion of ER $\alpha$ induces alterations in expression of some proteins involved in carbohydrate and lipid metabolism (75).

Whatsoever, estrogen actions in the heart seem to be more restricted to pathological states, during which estrogens mediate activation of mitochondrial biogenesis and cellular protection.

\section{Estrogen Effects on Skeletal Muscle Mitochondria}

Skeletal muscle possesses higher plasticity than cardiac muscle and exhibits considerable metabolic flexibility in response to hormonal stimulation, environmental factors, and exercise training. Skeletal muscles represent around $40 \%$ of body mass, and as a result they play a crucial role in insulin-sensitivity and glucose regulation.

Ovariectomy induces profound alteration of muscle biology and function. It decreases fatigue resistance, a factor known to depend on mitochondrial mass (76). Skeletal muscle of OVX mice shows lower use of fatty acid substrates, decreased PGC1 $\alpha$ expression, reduced mitochondrial content, and increased compensatory extramitochondrial ATP synthesis during exercise, 
most of which could be reverted by E2 treatment (77). Loss of E2 induces a rapid decline in skeletal muscle mitochondrial respiratory capacity, independently of mitochondrial content. This is likely mediated by changes in complex I function, accompanied by a $\sim 40 \%$ decrease in the reduced to oxidized glutathione ratio, effects that could also be reversed by E2 treatment (78).

Deletion of $\mathrm{ER} \alpha$ in skeletal muscle has profound metabolic consequences. Muscle-specific ER $\alpha$ knock-out mice (MERKO) exhibit decreased muscle mass, obesity, and insulin resistance with muscle mitochondrial dysfunction, altered mitochondrial dynamics and diminished mitophagy (79). Knock-down of ER $\alpha$ in $\mathrm{C} 2 \mathrm{C} 12$ myotubes reduces oxygen consumption rates and increased production of ROS thus promoting cellular oxidative stress (79). In parallel, the mammalian mitochondrial polymerase Polg1 is also down-regulated following deletion of ER $\alpha$, providing an additional mechanism for defective mitochondrial function in $E R \alpha$ deficiency. $E R \alpha$ also seems to affect mitochondrial quality control and dynamics because mitochondria of MERKO mouse muscle are elongated and hyperfused due to shift of the fusion/fission equilibrium toward fusion associated with a deficit in the fission protein DRP1 and increased levels of the fusion proteins OPA1 and MFN2 (79).

Conversely, estrogens are required to maintain muscle function because they have a positive effect on muscle mass, muscle regeneration after injury, and growth of satellite cells in young female mice [reviewed in (80)]. Estrogens regulate levels of enzymes associated with fatty acid metabolism explaining the higher capacity of woman skeletal muscles to utilize fatty acids $(81,82)$. In muscle cells, E2 plays also a major role in the inhibition of mitochondria-dependent apoptosis $(83,84)$. Physiological concentrations of E2 abrogate cytochrome $c$ release and DNA damage induced by oxidative stress (47).

The beneficial effects of E2 derive from both genomic and non-genomic effects. In $\mathrm{C} 2 \mathrm{C} 12$ muscle cells, E2 can interact with ERs located in the cell membrane and mitochondria to promote the activation of ERK, p38 MAPK, and the PI3K/Akt/pBad survival cascade thus abrogating the mitochondrial damage induced by hydrogen peroxide (83). E2 stimulates mitochondrial biogenesis in white gastrocnemius from OVX by increasing NRF1 transcript expression (85). Recently another mechanism by which E2 status broadly influences energy homeostasis has been described (78). It is shown that in skeletal muscle, E2 associates to the inner mitochondrial membrane, where it lowers membrane microviscosity, a mechanism that in turn promotes bioenergetic function, cellular redox balance, and insulin sensitivity thus offering a biophysical mechanism by which E2 influences energy homeostasis independently of ER $\alpha$ (78). This newly described mechanism of action of E2 on mitochondria may potentially be operative in other tissues; however, more studies are needed since this effect of E2 does not seem to be present in liver (86).

Importantly, postmenopausal women treated with hormone replacement therapy have substantially reduced relative risk of developing type 2 diabetes $(87,88)$. E2 exerts antidiabetogenic effects by protecting mitochondrial and cellular redox function in skeletal muscle of OVX mice (78). This insulin-sensitizing effect seems to be mediated by ER $\alpha$. Estrogens induce insulinsensitizing effects both by a reduction in the direct effects of ER $\alpha$ on insulin signaling as well as indirect effects of $\mathrm{ER} \alpha$ on insulin action mediated by mitochondrial dysfunction (89). Estrogens thus appear to be an important target allowing to correct metabolic dysfunctions by pharmacological means $(89,90)$.

\section{CONCLUSIONS AND PERSPECTIVES}

Estrogens are important participants in metabolic regulation and mitochondrial function. Mitochondria are involved in a vast range of cellular processes, and their dysfunction is intrinsically associated with chronic diseases. Accumulating evidence suggests that many chronic diseases display gender specificity with females generally exhibiting beneficial metabolic profile. Among possible factors, sex-specificity of mitochondria plays a major role in the sexual dimorphism of chronic pathologies (12). Some of these effects could be mediated by estrogens via estrogen receptors. Whether present in nucleus, in mitochondria or bound to cell membrane these latters exert profound genomic and nongenomic effects on mitochondria. They affect among other things mitochondrial biogenesis, ROS production, calcium homeostasis, and mitochondrial pathway of apoptosis in a tissue-specific manner.

Female sex is known to be associated with a reduced risk of developing cardiovascular diseases when compared to males $(15,91)$, an effect mediated in large part by estrogen and estrogen receptors. It appears that although sex differences in cardiovascular pathologies and metabolic diseases are being recognized, considerations of this issue in myopathologies are yet underestimated (13). Certainly, knowing the importance of mitochondria in the genesis of myopathologies, the sexual dimorphism of skeletal muscle mitochondria could be a contributing factor (13). However, our understanding of sex differences is still incomplete and highlights the importance of developing mechanistic studies to delineate more precisely estrogen receptor expression, localization, and function in males and female heart and skeletal muscles. It is still distressing that, while we are entering the era of personalized medicine and personalized therapeutic strategies, gender differences are still poorly taken into account in the majority of the experimental and clinical researches.

\section{AUTHOR CONTRIBUTIONS}

RV-C wrote the first version of the review. VV, AG, and JP participated actively in the writing of the final version.

\section{FUNDING}

$\mathrm{RV}-\mathrm{C}$ is emeritus scientist at CNRS. This work was supported by grants from Fondation de France (R18 199 LL) to AG.

\section{ACKNOWLEDGMENTS}

The authors wish to thank Véra Regitz-Zagrosek for her constant help and fruitful discussions. Figures have been drawn using Servier Medical Art (https://smart.servier.com/). 


\section{REFERENCES}

1. Calvo SE, Mootha VK. The mitochondrial proteome and human disease. Annu Rev Genomics Hum Genet. (2010) 11:25-44. doi: 10.1146/annurev-genom-082509-141720

2. Scarpulla RC, Vega RB, Kelly DP. Transcriptional integration of mitochondrial biogenesis. Trends Endocrinol Metab. (2012) 23:459-66. doi: 10.1016/j.tem.2012.06.006

3. Chen Y, Liu Y, Dorn GWn. Mitochondrial fusion is essential for organelle function and cardiac homeostasis. Circ Res. (2011) 109:1327-31. doi: 10.1161/CIRCRESAHA.111.258723

4. Ponsot E, Zoll J, N'guessan B, Ribera F, Lampert E, Richard R, et al. Mitochondrial tissue specificity of substrates utilization in rat cardiac and skeletal muscles. J Cell Physiol. (2005) 203:479-86. doi: 10.1002/jcp.20245

5. Saks V, Dzeja P, Schlattner U, Vendelin M, Terzic A, Wallimann T. Cardiac system bioenergetics: metabolic basis of Frank - Starling law. J Physiol. (2006) 571:253-73. doi: 10.1113/jphysiol.2005.101444

6. Kohlhaas M, Nickel AG, Maack C. Mitochondrial energetics and calcium coupling in the heart. J Physiol. (2017) 595:3753-63. doi: 10.1113/JP273609

7. Dzeja PP, Terzic A. Phosphotransfer networks and cellular energetics. J Exp Biol. (2003) 206:2039-47. doi: 10.1242/jeb.00426

8. Bernardi P, Di Lisa F. The mitochondrial permeability transition pore: molecular nature and role as a target in cardioprotection. J Mol Cell Cardiol. (2015) 78:100-6. doi: 10.1016/j.yjmcc.2014.09.023

9. Ventura-Clapier R, Kuznetsov A, Veksler V, Boehm E, Anflous K. Functional coupling of creatine kinases in muscles: species and tissue specificity. Mol Cell Biochem. (1998) 184:231-47. doi: 10.1007/978-1-4615-565 3-4_17

10. Ventura-Clapier R, Mettauer B, Bigard X. Beneficial effects of endurance training on cardiac and skeletal muscle energy metabolism in heart failure. Cardiovasc Res. (2007) 73:10-8. doi: 10.1016/j.cardiores.2006.09.003

11. Veksler VI, Kuznetsov AV, Anflous K, Mateo P, van Deursen J, Wieringa B, et al. Muscle creatine kinase-deficient mice.2. Cardiac and skeletal muscles exhibit tissue-specific adaptation of the mitochondrial function. J Biol Chem. (1995) 270:19921-9. doi: 10.1074/jbc.270.34.19921

12. Ventura-Clapier R, Moulin M, Piquereau J, Lemaire C, Mericskay M, Veksler $\mathrm{V}$, et al. Mitochondria: a central target for sex differences in pathologies. Clin Sci. (2017) 131:803-22. doi: 10.1042/CS20160485

13. Nye GA, Sakellariou GK, Degens H, Lightfoot AP. Muscling in on mitochondrial sexual dimorphism; role of mitochondrial dimorphism in skeletal muscle health and disease. Clin Sci. (2017) 131:1919-22. doi: $10.1042 /$ CS20160940

14. Vona R, Ascione B, Malorni W, Straface E. Mitochondria and sexspecific cardiac function. Adv Exp Med Biol. (2018) 1065:241-56. doi: 10.1007/978-3-319-77932-4_16

15. Regitz-Zagrosek V, and Kararigas G. Mechanistic pathways of sex differences in cardiovascular disease. Physiol Rev. (2017) 97:1-37. doi: 10.1152/physrev.00021.2015

16. Isensee J, Witt H, Pregla R, Hetzer R, Regitz-Zagrosek V, Ruiz Noppinger P. Sexually dimorphic gene expression in the heart of mice and men. J Mol Med. (2008) 86:61-74. doi: 10.1007/s00109-007-0240-z

17. Mayne BT, Bianco-Miotto T, Buckberry S, Breen J, Clifton V, Shoubridge $C$, et al. Large scale gene expression meta-analysis reveals tissuespecific, sex-biased gene expression in humans. Front Genet. (2016) 7:183. doi: 10.3389/fgene.2016.00183

18. Barba I, Miro-Casas E, Torrecilla JL, Pladevall E, Tejedor S, SebastianPerez R, et al. High-fat diet induces metabolic changes and reduces oxidative stress in female mouse hearts. J Nutr Biochem. (2017) 40:187-93. doi: 10.1016/j.jnutbio.2016.11.004

19. Sanz A, Hiona A, Kujoth GC, Seo AY, Hofer T, Kouwenhoven E, et al. Evaluation of sex differences on mitochondrial bioenergetics and apoptosis in mice. Exp Gerontol. (2007) 42:173-82. doi: 10.1016/j.exger.2006.10.003

20. Khalifa AR, Abdel-Rahman EA, Mahmoud AM, Ali MH, Noureldin M, Saber $\mathrm{SH}$, et al. Sex-specific differences in mitochondria biogenesis, morphology, respiratory function, and ROS homeostasis in young mouse heart and brain. Physiol Rep. (2017) 5:e13125. doi: 10.14814/phy2.13125

21. Vijay V, Han T, Moland CL, Kwekel JC, Fuscoe JC, Desai VG. Sexual dimorphism in the expression of mitochondria-related genes in rat heart at different ages. PLoS ONE. (2015) 10:e0117047. doi: 10.1371/journal.pone. 0117047

22. Yan L, Ge H, Li H, Lieber SC, Natividad F, Resuello RR, et al. Gender-specific proteomic alterations in glycolytic and mitochondrial pathways in aging monkey hearts. J Mol Cell Cardiol. (2004) 37:921-9. doi: $10.1016 /$ j.yjmcc.2004.06.012

23. Malorni W, Campesi I, Straface E, Vella S, Franconi F. Redox features of the cell: a gender perspective. Antioxid Redox Signal. (2007) 9:1779-801. doi: 10.1089 /ars.2007.1596

24. Lagranha CJ, Deschamps A, Aponte A, Steenbergen C, Murphy E. Sex differences in the phosphorylation of mitochondrial proteins result in reduced production of reactive oxygen species and cardioprotection in females. Circ Res. (2010) 106:1681-91. doi: 10.1161/CIRCRESAHA.109.213645

25. Ribeiro RF Jr, Ronconi KS, Morra EA, Do Val Lima PR, Porto ML, Vassallo DV, et al. Sex differences in the regulation of spatially distinct cardiac mitochondrial subpopulations. Mol Cell Biochem. (2016) 419:41-51. doi: 10.1007/s11010-016-2748-4

26. Chweih H, Castilho RF, Figueira TR. Tissue and sex specificities in Ca2+ handling by isolated mitochondria in conditions avoiding the permeability transition. Exp Physiol. (2015) 100:1073-92. doi: 10.1113/EP085248

27. Milerova M, Drahota Z, Chytilova A, Tauchmannova K, Houstek J, Ostadal B. Sex difference in the sensitivity of cardiac mitochondrial permeability transition pore to calcium load. Mol Cell Biochem. (2016) 412:147-54. doi: 10.1007/s11010-015-2619-4

28. Arieli Y, Gursahani H, Eaton MM, Hernandez LA, Schaefer S. Gender modulation of $\mathrm{Ca}(2+)$ uptake in cardiac mitochondria. J Mol Cell Cardiol. (2004) 37:507-13. doi: 10.1016/j.yjmcc.2004.04.023

29. De Bono JP, Adlam D, Paterson DJ, Channon KM. Novel quantitative phenotypes of exercise training in mouse models. Am J Physiol Regul Integr Comp Physiol. (2006) 290:R926-34. doi: 10.1152/ajpregu.00694.2005

30. Dworatzek E, Mahmoodzadeh S, Schubert C, Westphal C, Leber J, Kusch $A$, et al. Sex differences in exercise-induced physiological myocardial hypertrophy are modulated by oestrogen receptor beta. Cardiovasc Res. (2014) 102:418-28. doi: $10.1093 / \mathrm{cvr} / \mathrm{cvu} 065$

31. Wust RC, Morse CI, de Haan A, Jones DA, Degens H. Sex differences in contractile properties and fatigue resistance of human skeletal muscle. Exp Physiol. (2008) 93:843-50. doi: 10.1113/expphysiol.2007.041764

32. Devries MC. Sex-based differences in endurance exercise muscle metabolism: impact on exercise and nutritional strategies to optimize health and performance in women. Exp Physiol. (2016) 101:243-9. doi: 10.1113/EP085369

33. Tarnopolsky MA, Rennie CD, Robertshaw HA, Fedak-Tarnopolsky SN, Devries MC, Hamadeh MJ. The influence of endurance exercise training and sex on intramyocellular lipid and mitochondrial ultrastructure, substrate use, and mitochondrial enzyme activity. Am J Physiol Regul Integr Comp Physiol. (2006) 292:1271-8. doi: 10.1152/ajpregu.00472.2006

34. Colom B, Alcolea MP, Valle A, Oliver J, Roca P, Garcia-Palmer FJ. Skeletal muscle of female rats exhibit higher mitochondrial mass and oxidativephosphorylative capacities compared to males. Cell Physiol Biochem. (2007) 19:205-12. doi: 10.1159/000099208

35. Fernandez-Vizarra E, Enriquez JA, Perez-Martos A, Montoya J, FernandezSilva P. Tissue-specific differences in mitochondrial activity and biogenesis. Mitochondrion. (2011) 11:207-13. doi: 10.1016/j.mito.2010.09.011

36. Zoll J, Koulmann N, Bahi L, Ventura-Clapier R, Bigard AX. Quantitative and qualitative adaptation of skeletal muscle mitochondria to increased physical activity. J Cell Physiol. (2003) 194:186-93. doi: 10.1002/jcp. 10224

37. Cardinale DA, Larsen FJ, Schiffer TA, Morales-Alamo D, Ekblom B, et al. Superior intrinsic mitochondrial respiration in women than in men. Front Physiol. (2018) 9:1133. doi: 10.3389/fphys.2018.01133

38. Miotto PM, McGlory C, Holloway TM, Phillips SM, Holloway GP. Sex differences in mitochondrial respiratory function in human skeletal muscle. Am J Physiol Regul Integr Comp Physiol. (2018) 314:R909-15. doi: 10.1152/ajpregu.00025.2018

39. Montero D, Madsen K, Meinild-Lundby AK, Edin F, Lundby C. Sexual dimorphism of substrate utilization: differences in skeletal muscle mitochondrial volume density and function. Exp Physiol. (2018) 103:851-9. doi: 10.1113/EP087007 
40. Klinge CM. Estrogens regulate life and death in mitochondria. J Bioenergetics Biomembranes. (2017) 49:307-24. doi: 10.1007/s10863-017-9704-1

41. Ueda K, Karas RH. Emerging evidence of the importance of rapid, nonnuclear estrogen receptor signaling in the cardiovascular system. Steroids. (2013) 78:589-96. doi: 10.1016/j.steroids.2012.12.006

42. Lizotte E, Grandy SA, Tremblay A, Allen BG, Fiset C. Expression, distribution and regulation of sex steroid hormone receptors in mouse heart. Cell Physiol Biochem. (2009) 23:75-86. doi: 10.1159/000204096

43. Grohe C, Kahlert S, Lobbert K, Stimpel M, Karas RH, Vetter H, et al. Cardiac myocytes and fibroblasts contain functional estrogen receptors. FEBS Lett. (1997) 416:107-12. doi: 10.1016/S0014-5793(97)01179-4

44. Pugach EK, Blenck CL, Dragavon JM, Langer SJ, Leinwand LA. Estrogen receptor profiling and activity in cardiac myocytes. Mol Cell Endocrinol. (2016) 431:62-70. doi: 10.1016/j.mce.2016.05.004

45. Barros RP, Machado UF, Warner M, Gustafsson JA. Muscle GLUT4 regulation by estrogen receptors ERbeta and ERalpha. Proc Natl. Acad Sci USA. (2006) 103:1605-8. doi: 10.1073/pnas.0510391103

46. Wiik A, Hellsten Y, Berthelson P, Lundholm L, Fischer H, Jansson E. Activation of estrogen response elements is mediated both via estrogen and muscle contractions in rat skeletal muscle myotubes. Am J Physiol. (2009) 296:C215-20. doi: 10.1152/ajpcell.00148.2008

47. Vasconsuelo A, Milanesi L, Boland R. Actions of 17beta-estradiol and testosterone in the mitochondria and their implications in aging. Ageing Res Rev. (2013) 12:907-17. doi: 10.1016/j.arr.2013.09.001

48. Chen JQ, Cammarata PR, Baines CP, Yager JD. Regulation of mitochondrial respiratory chain biogenesis by estrogens/estrogen receptors and physiological, pathological and pharmacological implications. Biochim Biophys Acta. (2009) 1793:1540-70. doi: 10.1016/j.bbamcr.2009.06.001

49. Chen JQ, Delannoy M, Cooke C, Yager JD. Mitochondrial localization of ERalpha and ERbeta in human MCF7 cells. Am J Physiol Endocrinol Metab. (2004) 286:E1011-22. doi: 10.1152/ajpendo.00508.2003

50. Simpkins JW, Yang SH, Sarkar SN, Pearce V. Estrogen actions on mitochondria-physiological and pathological implications. Mol Cell Endocrinol. (2008) 290:51-9. doi: 10.1016/j.mce.2008.04.013

51. Yang SH, Liu R, Perez EJ, Wen Y, Stevens SMJ, Valencia T, et al. Mitochondrial localization of estrogen receptor beta. Proc Natl Acad Sci USA. (2004) 101:4130-5. doi: 10.1073/pnas.0306948101

52. Schwend T, Gustafsson JA. False positives in MALDI-TOF detection of ERbeta in mitochondria. Biochem Biophys Res Commun. (2006) 343:707-11. doi: 10.1016/j.bbrc.2006.02.164

53. Milanesi L, Russo de Boland A, Boland R. Expression and localization of estrogen receptor alpha in the $\mathrm{C} 2 \mathrm{C} 12$ murine skeletal muscle cell line. J Cell Biochem. (2008) 104:1254-73. doi: 10.1002/jcb.21706

54. Milanesi L, Vasconsuelo A, de Boland AR, Boland R. Expression and subcellular distribution of native estrogen receptor beta in murine C2C12 cells and skeletal muscle tissue. Steroids. (2009) 74:489-97. doi: 10.1016/j.steroids.2009.01.005

55. Vasconsuelo A, Milanesi L, Boland R. Participation of HSP27 in the antiapoptotic action of 17beta-estradiol in skeletal muscle cells. Cell Stress Chaperones. (2010) 15:183-92. doi: 10.1007/s12192-009-0132-y

56. Duckles SP, Krause DN, Stirone C, Procaccio V. Estrogen and mitochondria: a new paradigm for vascular protection? Mol Interven. (2006) 6:26-35. doi: 10.1124/mi.6.1.6

57. Yao J, Brinton RD. Estrogen regulation of mitochondrial bioenergetics: implications for prevention of Alzheimer's disease. Adv Pharmacol. (2012) 64:327-71. doi: 10.1016/B978-0-12-394816-8.00010-6

58. Rattanasopa C, Phungphong S, Wattanapermpool J, Bupha-Intr T. Significant role of estrogen in maintaining cardiac mitochondrial functions. J Steroid Biochem Mol Biol. (2015) 147:1-9. doi: 10.1016/j.jsbmb.2014.11.009

59. Velarde MC. Mitochondrial and sex steroid hormone crosstalk during aging. Longev Healthspan. (2014) 3:2. doi: 10.1186/2046-2395-3-2

60. Klinge CM. Estrogenic control of mitochondrial function and biogenesis. $J$ Cell Biochem. (2008) 105:1342-51. doi: 10.1002/jcb.21936

61. Witt H, Schubert C, Jaekel J, Fliegner D, Penkalla A, Tiemann K, et al. Sexspecific pathways in early cardiac response to pressure overload in mice. J Mol Med. (2008) 86:1013-24. doi: 10.1007/s00109-008-0385-4

62. Hsieh YC, Yang S, Choudhry MA, Yu HP, Rue LW III, Bland KI, et al. PGC-1 upregulation via estrogen receptors: a common mechanism of salutary effects of estrogen and flutamide on heart function after trauma-hemorrhage. Am J Physiol Heart Circ Physiol. (2005) 289:H2665-72. doi: 10.1152/ajpheart.00682.2005

63. Mattingly KA, Ivanova MM, Riggs KA, Wickramasinghe NS, Barch MJ, Klinge CM. Estradiol stimulates transcription of nuclear respiratory factor1 and increases mitochondrial biogenesis. Mol Endocrinol. (2008) 22:609-22. doi: 10.1210/me.2007-0029

64. Scarpulla RC. Nuclear control of respiratory gene expression in mammalian cells. J Cell Biochem. (2006) 97:673-83. doi: 10.1002/jcb.20743

65. Lancaster TS, Jefferson SJ, Hunter JC, Lopez V, Van Eyk JE, Lakatta EG, et al. Quantitative proteomic analysis reveals novel mitochondrial targets of estrogen deficiency in the aged female rat heart. Physiol Genomics. (2012) 44:957-69. doi: 10.1152/physiolgenomics.00184.2011

66. Meng Z, Jing H, Gan L, Li H, Luo B. Resveratrol attenuated estrogendeficient-induced cardiac dysfunction: role of AMPK, SIRT1, and mitochondrial function. Am J Transl Res. (2016) 8:2641-9.

67. Sbert-Roig M, Bauza-Thorbrugge M, Galmes-Pascual BM, Capllonch-Amer G, Garcia-Palmer FJ, Llado I, et al. GPER mediates the effects of 17betaestradiol in cardiac mitochondrial biogenesis and function. Mol Cell Endocrinol. (2016) 420:116-24. doi: 10.1016/j.mce.2015.11.027

68. Chen Y, Zhang Z, Hu F, Yang W, Yuan J, Cui J, et al. 17betaestradiol prevents cardiac diastolic dysfunction by stimulating mitochondrial function: a preclinical study in a mouse model of a human hypertrophic cardiomyopathy mutation. J Steroid Biochem Mol Biol. (2015) 147:92-102. doi: 10.1016/j.jsbmb.2014.12.011

69. Sun LY, Wang N, Ban T, Sun YH, Han Y, Sun LL, et al. MicroRNA23a mediates mitochondrial compromise in estrogen deficiency-induced concentric remodeling via targeting PGC-1alpha. J Mol Cell Cardiol. (2014) 75:1-11. doi: 10.1016/j.yjmcc.2014.06.012

70. Morkuniene R, Jekabsone A, Borutaite V. Estrogens prevent calcium-induced release of cytochrome c from heart mitochondria. FEBS Lett. (2002) 521:53-6. doi: 10.1016/S0014-5793(02)02820-X

71. Bopassa JC, Eghbali M, Toro L, Stefani E. A novel estrogen receptor GPER inhibits mitochondria permeability transition pore opening and protects the heart against ischemia-reperfusion injury. Am J Physiol Heart Circ Physiol. (2010) 298:H16-23. doi: 10.1152/ajpheart.00588.2009

72. Hsieh YC, Yu HP, Suzuki T, Choudhry MA, Schwacha MG, Bland KI, et al. Upregulation of mitochondrial respiratory complex IV by estrogen receptorbeta is critical for inhibiting mitochondrial apoptotic signaling and restoring cardiac functions following trauma-hemorrhage. J Mol Cell Cardiol. (2006) 41:511-21. doi: 10.1016/j.yjmcc.2006.06.001

73. Fliegner D, Schubert C, Penkalla A, Witt H, Kararigas G, Dworatzek E, et al Female sex and estrogen receptor-beta attenuate cardiac remodeling and apoptosis in pressure overload. Am J Physiol Regul Integr Comp Physiol. (2010) 298:R1597-606. doi: 10.1152/ajpregu.00825.2009

74. Kim JK, Pedram A, Razandi M, Levin ER. Estrogen prevents cardiomyocyte apoptosis through inhibition of reactive oxygen species and differential regulation of p38 kinase isoforms. J Biol Chem. (2006) 281:6760-7. doi: 10.1074/jbc.M511024200

75. Devanathan S, Whitehead T, Schweitzer GG, Fettig N, Kovacs A, Korach KS, et al. An animal model with a cardiomyocyte-specific deletion of estrogen receptor alpha: functional, metabolic, and differential network analysis. PLoS ONE. (2014) 9:e101900. doi: 10.1371/journal.pone.0101900

76. Nagai S, Ikeda K, Horie-Inoue K, Shiba S, Nagasawa S, Takeda S, et al. Estrogen modulates exercise endurance along with mitochondrial uncoupling protein 3 downregulation in skeletal muscle of female mice. Biochem Biophys Res Commun. (2016) 480:758-64. doi: 10.1016/j.bbrc.2016.10.129

77. Cavalcanti-de-Albuquerque JP, Salvador IC, Martins EL, Jardim-Messeder D, Werneck-de-Castro JP, Galina A, et al. Role of estrogen on skeletal muscle mitochondrial function in ovariectomized rats: a time course study in different fiber types. J Appl Physiol. (2014) 116:779-89. doi: 10.1152/japplphysiol.00121.2013

78. Torres MJ, Kew KA, Ryan TE, Pennington ER, Lin CT, Buddo KA, et al. 17beta-estradiol directly lowers mitochondrial membrane microviscosity and improves bioenergetic function in skeletal muscle. Cell Metab. (2018) 27:16779 e7. doi: 10.1016/j.cmet.2017.10.003

79. Ribas V, Drew BG, Zhou Z, Phun J, Kalajian NY, Soleymani T, et al. Skeletal muscle action of estrogen receptor alpha is critical for the maintenance of 
mitochondrial function and metabolic homeostasis in females. Sci Transl Med. (2016) 8:334ra54. doi: 10.1126/scitranslmed.aad3815

80. Ikeda K, Horie-Inoue K, Inoue S. Functions of estrogen and estrogen receptor signaling on skeletal muscle. J Steroid Biochem Mol Biol. (2019) 191:105375. doi: 10.1016/j.jsbmb.2019.105375

81. Ellis GS, Lanza-Jacoby S, Gow A, Kendrick ZV. Effects of estradiol on lipoprotein lipase activity and lipid availability in exercised male rats. J Appl Physiol. (1994) 77:209-15. doi: 10.1152/jappl.1994.77.1.209

82. Campbell SE, Febbraio MA. Effect of ovarian hormones on mitochondrial enzyme activity in the fat oxidation pathway of skeletal muscle. Am J Physiol Endocrinol Metab. (2001) 281:E803-8. doi: 10.1152/ajpendo.2001.281.4.E803

83. Boland R, Vasconsuelo A, Milanesi L, Ronda AC, de Boland AR. 17betaestradiol signaling in skeletal muscle cells and its relationship to apoptosis. Steroids. (2008) 73:859-63. doi: 10.1016/j.steroids.2007.12.027

84. Vasconsuelo A, Pronsato L, Ronda AC, Boland R, Milanesi L. Role of 17beta-estradiol and testosterone in apoptosis. Steroids. (2011) 76:1223-31. doi: 10.1016/j.steroids.2011.08.001

85. Capllonch-Amer G, Sbert-Roig M, Galmes-Pascual BM, Proenza AM, Llado I, Gianotti M, et al. Estradiol stimulates mitochondrial biogenesis and adiponectin expression in skeletal muscle. J Endocrinol. (2014) 221:391-403. doi: 10.1530/JOE-14-0008

86. Torres MJ, Ryan TE, Lin CT, Zeczycki TN, Neufer PD. Impact of $17 \beta$-estradiol on complex I kinetics and $\mathrm{H}_{2} \mathrm{O}_{2}$ production in liver and skeletal muscle mitochondria. J Biol Chem. (2018) 293:16889-98. doi: $10.1074 /$ jbc.RA118.005148

87. Salpeter SR, Walsh JM, Ormiston TM, Greyber E, Buckley NS, Salpeter EE. Meta-analysis: effect of hormone-replacement therapy on components of the metabolic syndrome in postmenopausal women. Diabetes Obes Metab. (2006) 8:538-54. doi: 10.1111/j.1463-1326.2005.00545.x
88. Pentti K, Tuppurainen MT, Honkanen R, Sandini L, Kroger H, Alhava E, et al. Hormone therapy protects from diabetes: the Kuopio osteoporosis risk factor and prevention study. Eur J Endocrinol. (2009) 160:979-83. doi: 10.1530/EJE-09-0151

89. Hevener AL, Zhou Z, Moore TM, Drew BG, Ribas V. The impact of ERalpha action on muscle metabolism and insulin sensitivity - Strong enough for a man, made for a woman. Mol Metab. (2018) 15:20-34. doi: 10.1016/j.molmet.2018.06.013

90. Gupte AA, Pownall HJ, Hamilton DJ. Estrogen: an emerging regulator of insulin action and mitochondrial function. J Diabetes Res. (2015) 2015:916585. doi: $10.1155 / 2015 / 916585$

91. EUGenMed Cardiovascular Clinical Study, Regitz-Zagrosek V, OerteltPrigione S, Prescott E, Franconi F, Gerdts E, et al. Gender in cardiovascular diseases: impact on clinical manifestations, management, and outcomes. Eur Heart J. (2016) 37:24-34. doi: 10.1093/eurheartj/ehv598

Conflict of Interest Statement: The authors declare that the research was conducted in the absence of any commercial or financial relationships that could be construed as a potential conflict of interest.

The handling Editor declared a past co-authorship with one of the authors RV-C.

Copyright (๑ 2019 Ventura-Clapier, Piquereau, Veksler and Garnier. This is an open-access article distributed under the terms of the Creative Commons Attribution License (CC BY). The use, distribution or reproduction in other forums is permitted, provided the original author(s) and the copyright owner(s) are credited and that the original publication in this journal is cited, in accordance with accepted academic practice. No use, distribution or reproduction is permitted which does not comply with these terms. 